\title{
Study of anomalous tau lepton decay using chiral Lagrangian with vector mesons
}

\author{
Takuya Morozumi*, Hiroyuki Umeeda \\ Graduate School of Science, Hiroshima University, Higashi-Hiroshima 739-8526, Japan \\ Core of Research for the Energetic Universe, Hiroshima University, Higashi-Hiroshima \\ 739-8526, Japan \\ E-mail: \\ morozumi@hiroshima-u.ac.jp, umeeda@theo.phys.sci.hiroshima-u.ac.jp
}

\section{Daiji Kimura}

General Education, Ube National College of Technology, Ube, Yamaguchi 755-8555, Japan

E-mail: kimuradeube-k.ac.jp

\begin{abstract}
An intrinsic parity violating hadronic tau lepton decay is investigated. $\tau \rightarrow \pi \pi \eta v$ is the process in which the dominant contribution to the amplitude is due to the intrinsic parity violation. To predict the hadronic invariant mass spectra and to compare them with experimental data, we extend the chiral Lagrangian with vector mesons so that it incorporates the intrinsic parity violating terms and $\phi$ and $\eta^{\prime}$ mesons. The coefficients of the intrinsic parity violating terms will be determined by fitting the branching fractions for $V^{I} \rightarrow P \gamma, V^{I} \rightarrow 3 P$ and $P \rightarrow V^{I} \gamma$ where $V^{I}$ denotes vector mesons $1^{-}$and $P$ denotes pseudo-scalar mesons $0^{-}$.
\end{abstract}

Flavor Physics and CP Violation 2015

May 25-29, 2015

Nagoya, Japan

${ }^{*}$ Speaker. 


\section{Introduction}

Hadronic tau decays are important because the precise predictions for the process lead to the understanding QCD of energy range from $1(\mathrm{GeV})$ up to $2(\mathrm{GeV})$. In this energy region, there are many resonances and the description of the chiral perturbation including only SU(3) octet pseudo-scalar mesons is not sufficient. Since there are many resonances, perturbative QCD is not applicable. Though there are also many resonance models with which the hadronic form factors are computed, the theoretical framework which can be systematically improved has been waited for. Incorporating light $\mathrm{SU}(3)$ octet vector mesons, a framework which enables us to compute the quantum corrections of pseudo Nambu-Goldstone bosons in a systematic way, has been proposed [1]. In the previous work [1], $\tau \rightarrow K \pi \nu$ decay is studied and the vector and scalar form factors are computed. As for the vector mesons, $K^{*}$ meson contributes to the form factors. The aim of our study is to extend the Lagrangian including intrinsic parity violation. In the hadronic tau decay including more than two pseudo-scalars as final states of hadrons, the intrinsic parity violation is very important [2]. For instance, in the hadronic tau decays with final states of odd number of pseudo-scalars plus a neutrino, the vector current contribution is intrinsic parity violating while in the process with the final states with even number of pseudo-scalars plus a neutrino, the axial current contribution is intrinsic parity violating.

\section{2. $\tau^{-} \rightarrow \pi^{-} \pi^{0} \eta v$}

In the decay $\tau^{-} \rightarrow \pi^{-} \pi^{0} \eta v$, the intrinsic parity violating contribution is dominant contribution, because intrinsic parity conserving contribution arises as axial current contribution and it is suppressed due to $\mathrm{G}$ parity. The matrix elements of vector form factor $\left\langle\pi^{0} \pi^{-} \eta\left|\bar{d} \gamma_{\mu} u\right| 0\right\rangle$ is intrinsic parity violating. Wess Zumino term [3] which represents chiral anomaly contributes to the matrix element. However, the other intrinsic parity violating terms which include the vector mesons also contribute to the process. As one can see from the Feynman diagrams shown in Fig.1, there are many intrinsic parity violating vertices which contribute to the decay. They are $V \rightarrow \pi^{-} \pi^{0} \eta$, $V \rightarrow \rho \eta$ and $V \rightarrow \rho \pi$ where we denote $V$ as weak gauge boson. These vertices are related to $\gamma^{*} \rightarrow 3 P, V^{I} \rightarrow P \gamma$, where we denote $V^{I}$ as vector mesons and $P$ as pseudo-scalars. One also has vertices like $\rho^{-} \rightarrow \rho^{-} \eta, \rho^{-} \rightarrow \rho^{-} \pi^{0}, \rho^{-} \rightarrow \pi^{-} \pi^{0} \eta$ and they are related to the processes like $V^{I} \rightarrow V^{J} P$ and $V^{I} \rightarrow 3 P$.

\section{Chiral Lagrangian with vector mesons with intrinsic parity violation}

Our aim is to extend the chiral Lagrangian and include the intrinsic parity violating terms. The intrinsic parity violating terms besides Wess Zumino term can be written as follows.

$$
\begin{aligned}
& \mathscr{L}_{1}=i \varepsilon^{\mu v \rho \sigma} \operatorname{Tr}\left[\alpha_{L \mu} \alpha_{L v} \alpha_{L \rho} \alpha_{R \sigma}-(R \leftrightarrow L)\right], \\
& \mathscr{L}_{2}=i \varepsilon^{\mu v \rho \sigma} \operatorname{Tr}\left[\alpha_{L \mu} \alpha_{R v} \alpha_{L \rho} \alpha_{R \sigma}\right], \\
& \mathscr{L}_{3}=-\frac{1}{2} \varepsilon^{\mu v \rho \sigma} \operatorname{Tr}\left[F_{V \mu v}\left\{\alpha_{L \rho} \alpha_{R \sigma}-(R \leftrightarrow L)\right\}\right], \\
& \mathscr{L}_{4}=\varepsilon^{\mu v \rho \sigma} \operatorname{Tr}\left[\left(\hat{F}_{L \mu v}+\hat{F}_{R \mu v}\right)\left\{\alpha_{L \rho}, \alpha_{R \sigma}\right\}\right],
\end{aligned}
$$




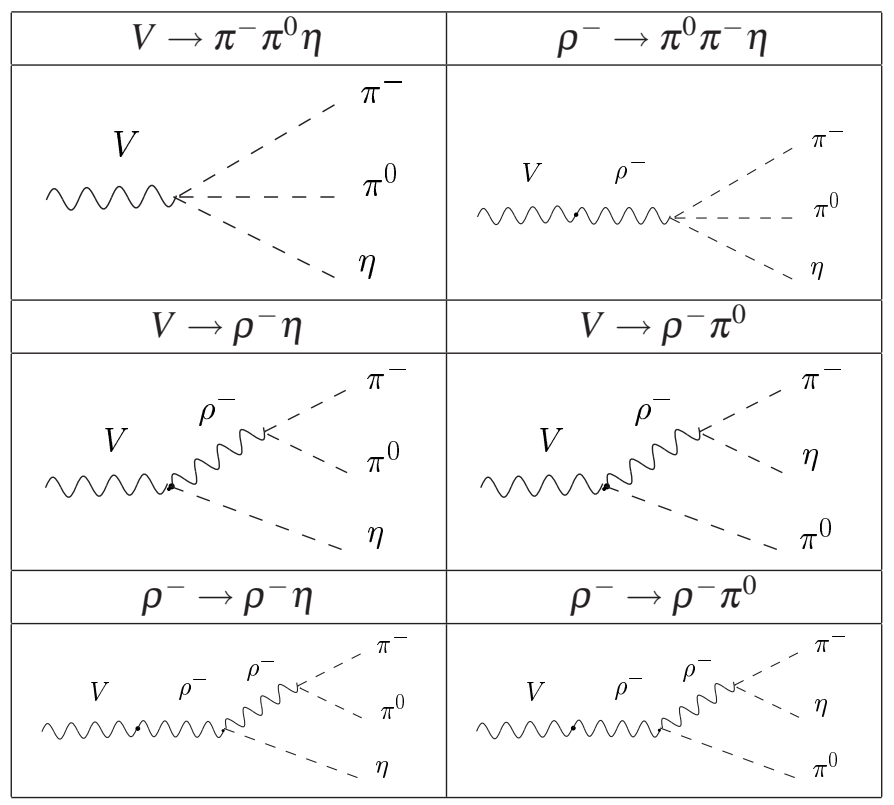

Figure 1: The Feynman diagrams which contribute to the vector form factor of $\tau \rightarrow \pi^{-} \pi^{0} \eta v$. V denotes the external vector current part of $V-A$ charged current, $V_{\mu}=\bar{d} \gamma_{\mu} u$.

$$
\begin{aligned}
& \mathscr{L}_{5}=\varepsilon^{\mu v \rho \sigma} F_{V \mu \nu}^{0} \operatorname{Tr}\left[\alpha_{L \rho} \alpha_{R \sigma}-(R \leftrightarrow L)\right], \\
& \mathscr{L}_{6}=\frac{\eta_{0}}{f} \varepsilon^{\mu v \rho \sigma} \operatorname{Tr} F_{V \mu \nu} F_{V \rho \sigma}, \\
& \mathscr{L}_{7}=\frac{\eta_{0}}{f} \varepsilon^{\mu v \rho \sigma} F_{V \mu v}^{0} F_{V \rho \sigma}^{0} .
\end{aligned}
$$

In Eqs.(3.1)-(3.7), $\alpha_{L}$ and $\alpha_{R}$ include pseudo-scalar octet. $F_{L \mu v}$ and $F_{R \mu v}$ are field strength for gauge bosons. $F_{V \mu \nu}$ is a field strength of SU(3) octet vector mesons. $F_{\mu \nu}^{0}$ denotes the field strength of SU(3) singlet vector meson $\phi^{0}$. Neutral components of octet and singlet vector mesons form $\rho$, $\phi$ and $\omega$ mesons through their mixing. The Lagrangian of intrinsic parity violating terms is given as,

$$
\mathscr{L}=\sum_{i=1}^{7} c_{i I P V} \mathscr{L}_{i}
$$

$\mathscr{L}_{1,2,3}$ given in Eqs.(3.1)-(3.3) are introduced in Refs.[4] and [5]. We newly consider the operator in Eqs.(3.5)-(3.7). In Eqs.(3.1)-(3.7), we also required that the operators should be Hermite. The Lagrangian is invariant under chiral $\mathrm{SU}(3) \times \mathrm{SU}(3)$. The coefficients can be constrained using $V^{I} \rightarrow P \gamma, P \rightarrow \gamma \gamma$ and $V^{I} \rightarrow 3 P$. Once the coefficients are fixed, one can apply it to the form factor calculation of $\tau$ decay. Alternatively, one can use the experimental distributions for the decay [6] to determine the coefficients.

\section{The vector meson propagator and a consistent treatment of vector mesons and $\gamma$ mixing in $V^{I} \rightarrow P^{i} \gamma$ amplitude}

As shown in Fig.1, $\rho^{-}$meson contributes to the process. In the distribution with respect to 

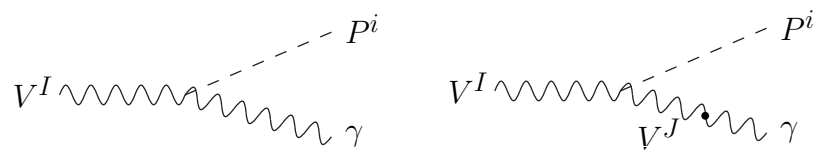

Figure 2: The Feynman diagrams for $V^{I} \rightarrow P^{i} \gamma$ decay. The diagram of the right includes intermediate vector meson propagator.

invariant mass such as $m_{\pi^{-}} \pi^{0}$ and $m_{\pi^{-}} \eta \rho^{-}$should show up as the resonance. In our framework, the $\rho^{-}$meson propagator is obtained by calculating one-loop corrections to the self energy of the vector mesons due to pseudo-scalars. In the process $V^{I} \rightarrow P^{i} \gamma$ in Fig.2, the left panel does not include the vector meson propagator. In the diagram of the right panel, the neutral vector meson propagator is included. The neutral vector mesons correspond to $\rho^{0}, \omega$ and $\phi$ and they mix with photon in the final states. Precision of the calculation requires us to use one -loop corrected vector meson propagator as in the case of the vector form factor of tau decay. The naive use of the one loop corrected propagator in $V^{I} \rightarrow P^{i} \gamma$ decay leads to the result which depends on the propagators of intermediate vector mesons denoted by $V^{J}$ in Fig.2,

$$
D_{V^{J}}\left(q_{\gamma}^{2}=0\right),
$$

where $q_{\gamma}$ is a four momentum of the photon. $q_{\gamma}^{2}=0$ implies the vector mesons $V^{J}$ mix with on-shell photon $\left(q_{\gamma}^{2}=0\right)$. To be consistent, one must use the one-loop corrected $\gamma$ and vector mesons' mixing. By using it, one can show the amplitude does not depend on the propagator of the intermediate vector meson at all.

\section{References}

[1] D. Kimura, K. Y. Lee and T. Morozumi, PTEP 2013, 053 B03 (2013) [Erratum-ibid. 2014, no. 8, 089202 (2014)] [arXiv:1201.1794 [hep-ph]].

[2] A. Pich, Phys. Lett. B 196, 561 (1987).

[3] J. Wess and B. Zumino, Phys. Lett. B 37, 95 (1971).

[4] T. Fujiwara, T. Kugo, H. Terao, S. Uehara and K. Yamawaki, Prog. Theor. Phys. 73, 926 (1985).

[5] M. Bando, T. Kugo and K. Yamawaki, Phys. Rept. 164, 217 (1988).

[6] K. Inami et al. [Belle Collaboration], Phys. Lett. B 672, 209 (2009). 